\title{
Self-reported Sleep Quality and Bone Outcomes in Older Adults: Findings from the Hertfordshire Cohort Study
}

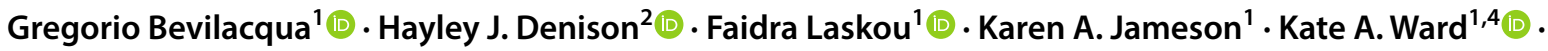 \\ Cyrus Cooper $^{1,3}$ (Elaine M. Dennison ${ }^{1,5}$ (1)
}

Received: 28 October 2019 / Accepted: 7 January 2020 / Published online: 18 January 2020

(c) The Author(s) 2020

\begin{abstract}
Sleep duration may be associated with risk of osteoporosis, with suggestions that too little or indeed too much sleep may be detrimental to bone health. In this study, we considered whether perceived sleep quality is also associated with bone health in older adults. We explored this association in a cohort of 443 older community-dwelling UK adults. Sleep quality was assessed using the Pittsburgh Sleep Quality Index (PSQI); poor sleep quality was defined as $>5$ on this score system. Bone density, shape and microarchitecture were assessed using dual energy X-ray absorptiometry (DXA), peripheral quantitative computed tomography (pQCT) and high-resolution pQCT (HRpQCT). Thirty-seven percent of men and 43\% of women had a PSQI score greater than 5, indicative of poor perceived sleep. We found that quality of sleep was associated with altered bone microarchitecture. In men, poor sleep quality was associated with lower radial trabecular $(4 \%$ slice, $p<0.04)$ and cortical $(66 \%$ slice, $p=0.02)$ bone mineral density, as well as decreased tibial cortical density $(p<0.02)$ and increased porosity $(p<0.04)$, but increased size of the tibia $(p<0.04)$. In women, poor perceived sleep quality was associated with thinner $(p<0.03)$ and less dense $(p<0.04)$ cortices of the radius, but greater tibial trabecular number $(p<0.02)$ and lower separation $(p<0.04)$. Relationships with DXA parameters were non-significant after adjustment for confounders. Taking sleep medications was associated with decreased tibial size (38\% and $66 \%$ slices) and strength in women (all $p<0.05$ ), but not in men. Perceived sleep quality was associated with altered bone density and microarchitecture in older adults, and these differences varied according to biological sex and site. Further work is indicated to investigate possible mechanisms underlying these observations.
\end{abstract}

Keywords Sleep quality $\cdot$ Older adults $\cdot$ Bone microarchitecture $\cdot$ Computed tomography $\cdot$ Ageing $\cdot$ Bone density

\section{Introduction}

Gregorio Bevilacqua

gb@mrc.soton.ac.uk

1 MRC Lifecourse Epidemiology Unit, University of Southampton, Southampton General Hospital, Southampton SO16 6YD, UK

2 Centre for Public Health Research, Massey University, Wellington, New Zealand

3 National Institute for Health Research Musculoskeletal Biomedical Research Unit, University of Oxford, Oxford, UK

4 MRC Nutrition and Bone Health Group, Cambridge, UK

5 Victoria University of Wellington, Wellington, New Zealand
Sleep quality represents a fundamental aspect of optimal health and functioning [1]. Sleep experience can be highly subjective and related to different aspects, such as sleep onset period and frequent awakenings [2]. Sleep quality has been proven to decrease with age [3], and insufficient sleep and sleep disorder have been associated with adverse outcomes such as weight gain and obesity, diabetes, inflammation, cardiovascular disease, neurocognitive health, mental health and mortality, and these relationships have been widely discussed in the media [4]. It has also been theorised that sleep duration may be associated with risk of osteoporosis, with previous studies suggesting that insufficient or indeed excessive sleep might affect bone health in middle-aged and elderly adults [5]. However, previous studies have focussed mostly on short sleep duration [5-10] 
and obstructive sleep apnoea [11-16] as possible contributors to osteoporosis.

In the current study, we sought to explore, in a cohort of community-dwelling older adults in the UK, whether perceived sleep quality was associated with bone health and microarchitecture, and whether the relationships were the same in men and women.

\section{Methods}

Participants were recruited from the Hertfordshire Cohort Study (HCS), a population-based sample of men and women born between 1931 and 1939 in Hertfordshire and originally recruited in order to study the relationship between growth in infancy and the subsequent risk of adult diseases [17, 18]. Participants completed a nurse-administered questionnaire in 1998-2002, which included details of socioeconomic status, lifestyle and diet, including calcium intake.

In 2011-2012, 443 HCS participants [222 men and 221 women; mean (SD) age 75.5 (2.5) years for men and 75.8 (2.6) for women] were visited at home by a trained fieldworker, who administered a lifestyle questionnaire. Participants were also provided with a self-administered questionnaire, which was returned to the researchers at the MRC Lifecourse Epidemiology Unit. This self-administered questionnaire included a sleep assessment tool. We assessed sleep quality using the Pittsburgh Sleep Quality Index (PSQI), a validated tool for assessing the quality of a person's sleep; it provides a measure of sleep quality based on an individual's evaluation of a range of sleep measures over the time period of one month [19]. The PSQI questionnaire consists of seven subcomponents: subjective sleep quality, sleep latency, sleep duration, habitual sleep efficiency, sleep disturbances, use of sleeping medication and daytime dysfunction. Each domain is weighted on a 0-3 interval scale. Answers are used to generate individual scores for each of the components, and by summing the subcomponents' scores, a global PSQI score (ranging 0-21) is generated. A score greater than 5 is indicative of poor sleep quality and has been proven to have high sensitivity and specificity in distinguishing good and poor sleepers [19]. The PSQI questionnaire was returned by 401 (91\%) of the participants (201 men and 200 women). We also assessed the prevalence of comorbid diseases in our study population. These were self-reported by participants via questionnaire, asking the question: 'Have you been told by a doctor that you have any of the following conditions?' The following comorbidities were recorded: hypertension, heart disease, stroke, diabetes, lung disease, thyroid disease, rheumatoid arthritis, multiple sclerosis, vitiligo, depression, Parkinson's disease, peripheral arterial disease, osteoporosis and cancer. Social class was determined from the participants own current or most recent occupation for men and never-married women and of the husband for married women and was classified as non-manual (classes I-IIINM) or manual (classes IIIM-V) according to the 1990 OPCS Standard Occupational Classification scheme. Physical activity was calculated as the average minutes per day spent in walking, cycling, gardening, playing sport and doing house work in the last 2 weeks.

In the same year, participants attended a research clinic where the following tests were performed: a dual X-ray absorptiometry (DXA) scan (Lunar Prodigy Advanced Scanner, GE Medical Systems, UK), a peripheral quantitative computed tomography (pQCT, Stratec XCT2000, Stratec Medizintechnik, Germany) scan and a high-resolution pQCT (HRpQCT, XTreme I, Scanco, Switzerland) scan of the nondominant radius and tibia (except when the non-dominant limb had previously fractured, in which case the dominant side was scanned). All scans were acquired by a trained technician using standard positioning techniques. Of those invited, 376 men and women agreed to participate [20].

DXA scans allowed us to measure bone mineral content (g) and areal bone mineral density $\left(\mathrm{g} / \mathrm{cm}^{2}\right)$ at the non-dominant hip. Positioning for all scans was completed in accordance with the manufacturer's instructions. For pQCT scans, the radial length was measured from the distal end of the ulna styloid to the tip of the olecranon in millimetres ( $\mathrm{mm})$. The tibial length was measured from the prominence of the medial malleolus to the tibial plate $(\mathrm{mm})$. Radial and tibial scout views identified measurement reference lines at the cortical end plates. Two slices were taken from the radial scan (4 and 66\%). Four slices were taken from the tibial scan $(4,14,38$, and $66 \%)$. Trabecular parameters were measured distally (4\% radius and $4 \%$ tibia) and cortical, muscle and subcutaneous fat parameters were measured in the midshaft $(66 \%)$ for the radius and in both the mid-shaft (4\% and $38 \%$ ) and proximal tibia (66\%). The following measurements were taken from the radius and tibia: total bone area (Tt.Ar), total mass, trabecular bone mineral density (tBMD), cortical bone mineral density (cBMD), cortical bone area ( $\mathrm{Ct}$.Ar), polar strength strain index (SSI) and fracture load. Shortterm measurement precision error ranged from $0.88 \%$ (total tibial density, $4 \%$ slice) to $8.8 \%$ (total radial area, $66 \%$ slice), but was typically between 1 and $3 \%$. These figures were obtained by 20 volunteers who were part of the study undergoing two scans on the same day, with limb repositioning between examinations. For all scans, a threshold of $280 \mathrm{mg} /$ $\mathrm{cm}^{3}$ was used to separate the bone from the soft tissue background. Once separated, the default peeling algorithm was applied to the distal $4 \%$ scans to separate trabecular bone. With this peeling, $55 \%$ of the outer bone area was concentrically separated and defined as cortical and subcortical; the remaining $45 \%$ was defined as trabecular bone. For proximal 
scan locations, the default threshold of $710 \mathrm{mg} / \mathrm{cm}^{3}$ was used to separate cortical bone. A threshold of $280 \mathrm{mg} / \mathrm{cm}^{3}$ was used for polar SSI $\left(\mathrm{mm}^{3}\right)$ calculation.

HRpQCT scans consist of a stack of 110 parallel CT slices using a two-dimensional detector array; the volume of interest is $9 \mathrm{~mm}$ in axial length with a nominal resolution (voxel size) of $82 \mu \mathrm{m}$. The scanned limb was immobilized with a carbon fibre cast during the examination. Anteroposterior $2 \mathrm{D}$ scout views were performed to determine the region to be scanned. Positioning was in keeping with the manufacturer's guidelines and as described by Boutroy et al. [21]. Each scan was assessed for motion artefact, and, if present, a second scan was performed. A total of 40 radial images and 9 tibial images were excluded due to excessive motion artefact. Initial image analysis was carried out using the standard manufacturer's method and Image Processing Language (IPL, Version 6.1, ScancoMedical). Further analysis was performed using an automated segmentation algorithm to obtain cortical porosity parameters. Each measure used has been validated against micro-CT imaging.

\section{Statistical Analysis}

Descriptive statistics for continuous variables were expressed as mean and standard deviation (SD) or median and interquartile range (IQR) as appropriate. Categorical variables were expressed as frequency and percentage. Differences between men and women were assessed using Student's $t$-tests, Mann-Whitney $U$ tests, Pearson's $\chi^{2}$ tests or Fisher's exact tests, as appropriate. The bone outcomes were transformed to Fisher-Yates (FY) $z$-scores using the Fisher-Yates rank-based inverse normal transformation to normalise the data. Linear regression analyses were used to examine the associations between poor sleep quality (PSQI > 5) and the PSQI subcomponents and bone outcomes. The regression analyses were undertaken with and without adjusting for the following confounders: age, BMI, social class, smoker status (never, ex-smoker or current smoker), alcohol consumption (units per week), physical activity, dietary calcium intake [22, 23], number of comorbidities and, in women, years since menopause and hormone replacement therapy (HRT) use. The results of the regression analyses are presented as regression coefficients $(\beta)$ and $95 \%$ confidence intervals (CI). A $p$-value of $\leq 0.05$ was considered to be statistically significant. The analyses were conducted using Stata version 14.

\section{Results}

Data on quality of sleep, BMD and bone microarchitecture were available for 323 participants, of whom 169 were men and 154 women. Table 1 provides the demographic characteristics of the participants included in the study. The mean (SD) age of participants in the study was 75.4 (2.5) years for men and 75.7 (2.6) years for women, with the mean BMI differing little between the sexes. More significant sex differences were observed in alcohol consumption, daily dietary calcium intake and smoking habits: men consumed more alcohol per week than women (median (IQR): 6.9 units (1.8-14.0) for men, 0.5 units (0.0-4.5) for women), had a higher daily dietary calcium intake than women (median (IQR): $1233 \mathrm{mg}$ (1011-1413) for men, $1087 \mathrm{mg}$ (913-1254) for women), and were more prone to be or have been a smoker than women, with $41.4 \%$ of them reporting to have never smoked, whereas $63.6 \%$ of women said they had never smoked. Comorbidity affected men more than women: $24 \%$ of female participants reported having no comorbid disease, while $19.5 \%$ of men said they did not have any of the conditions listed. On the other hand, more women (10.4\%) than men $(7.1 \%)$ reported having 4 or more concurrent diseases. Social class distribution was similar in both sexes. The majority of women $(55.1 \%)$ reported never having used HRT, with only $2.7 \%$ of them saying that they were currently using HRT.

The median (IQR) PSQI score was 4 (2-7) for men and 5 (3-8) for women. Thirty-seven percent of men and $43 \%$ of women had a PSQI score greater than 5, indicative of poor reported sleep quality. The mean (SD) sleep duration was 6.9 (1.1) hours per night for men and women 6.9 (1.2) hours per night for women.

PSQI overall score and individual PSQI subcomponents showed no significant association with DXA-derived areal BMD, following adjustment for confounders. Conversely, relationships were found between a PSQI score $>5$ and several of the bone outcomes assessed by pQCT and HRpQCT. The associations between poor quality of sleep and bone outcomes as measured by pQCT and HRpQCT for both men and women are presented in Figs. 1 (radius) and 2 (tibia). In men, poor sleep quality was associated with lower radial mass $(\beta-0.32 z$-score, 95\% CI $-0.64,-0.00, p<0.05)$ and tBMD $(\beta-0.34 z$-score, 95\% CI $-0.65,-0.02, p<0.05)$ at $4 \%$ slice, and lower radial cBMD $(\beta-0.38 z$-score, $95 \% \mathrm{CI}-0.70,-0.06, p<0.02)$ at $66 \%$ slice. The associations between poor sleep and these pQCT outcomes, with the exception of radial mass at $4 \%$ slice, remained significant following adjustment for confounders. We also found associations between PSQI score $>5$ and HRpQCT outcomes in men: in particular, poor sleep was associated with lower radial trabecular thickness $(\beta-0.41 z$-score, $95 \%$ $\mathrm{CI}-0.75,-0.06, p<0.03)$, higher tibial trabecular area $(\beta$ $0.37 z$-score, $95 \%$ CI $0.05,0.69, p<0.03$ ) and lower tibial cBMD ( $\beta-0.35 z$-score, 95\% CI $-0.67,-0.04, p=0.03$ ) and apparent cortical thickness $(\beta-0.34 z$-score, $95 \% \mathrm{CI}$ $-0.66,-0.02, p<0.04)$. Following adjustment for confounders, the associations with radial trabecular thickness and 
Table 1 Baseline characteristics of study participants by biological sex

\begin{tabular}{|c|c|c|c|c|c|c|c|}
\hline & \multicolumn{3}{|l|}{ Men } & \multicolumn{3}{|l|}{ Women } & \multirow[t]{2}{*}{$p$-value } \\
\hline & $N$ & Mean & SD & $N$ & Mean & SD & \\
\hline Age (years) & 169 & 75.4 & 2.5 & 154 & 75.7 & 2.6 & 0.441 \\
\hline \multirow[t]{2}{*}{ BMI $\left(\mathrm{kg} / \mathrm{m}^{2}\right)$} & 169 & 27.7 & 3.8 & 152 & 28.3 & 4.9 & 0.174 \\
\hline & $N$ & Median & IQR & $N$ & Median & IQR & $p$-value \\
\hline Activity time in last 2 weeks (min/day) & 156 & 187 & $127-274$ & 146 & 206 & $141-283$ & 0.267 \\
\hline Daily dietary calcium intake (mg) & 169 & 1233 & $1011-1413$ & 154 & 1087 & $913-1254$ & $<0.001$ \\
\hline \multirow[t]{2}{*}{ Alcohol consumption (units per week) } & 169 & 6.9 & $1.8-14.0$ & 154 & 0.5 & $0.0-4.5$ & $<0.001$ \\
\hline & Total $N$ & $N$ & $\%$ & Total $N$ & $N$ & $\%$ & $p$-value \\
\hline Smoker status & 169 & & & 154 & & & $<0.001$ \\
\hline Never & & 70 & 41.4 & & 98 & 63.6 & \\
\hline Ex & & 93 & 55.0 & & 52 & 33.8 & \\
\hline Current & & 6 & 3.6 & & 4 & 2.6 & \\
\hline Social class & 162 & & & 154 & & & 0.958 \\
\hline I-IIINM & & 70 & 43.2 & & 67 & 43.5 & \\
\hline IIIM-V & & 92 & 56.8 & & 87 & 56.5 & \\
\hline No. of comorbidities & 169 & & & 154 & & & 0.388 \\
\hline 0 & & 33 & 19.5 & & 37 & 24.0 & \\
\hline 1 & & 66 & 39.1 & & 45 & 29.2 & \\
\hline 2 & & 41 & 24.3 & & 39 & 25.3 & \\
\hline 3 & & 17 & 10.1 & & 17 & 11.0 & \\
\hline \multirow[t]{2}{*}{$\geq 4$} & & 12 & 7.1 & & 16 & 10.4 & \\
\hline & $N$ & Median & IQR & $N$ & Median & IQR & $p$-value \\
\hline \multirow[t]{2}{*}{ PSQI score } & 156 & 4 & $2-7$ & 144 & 5 & $3-8$ & 0.033 \\
\hline & Total $N$ & $N$ & $\%$ & Total $N$ & $N$ & $\%$ & $p$-value \\
\hline PSQI score > 5 & 156 & 58 & 37.2 & 144 & 62 & 43.1 & 0.346 \\
\hline
\end{tabular}
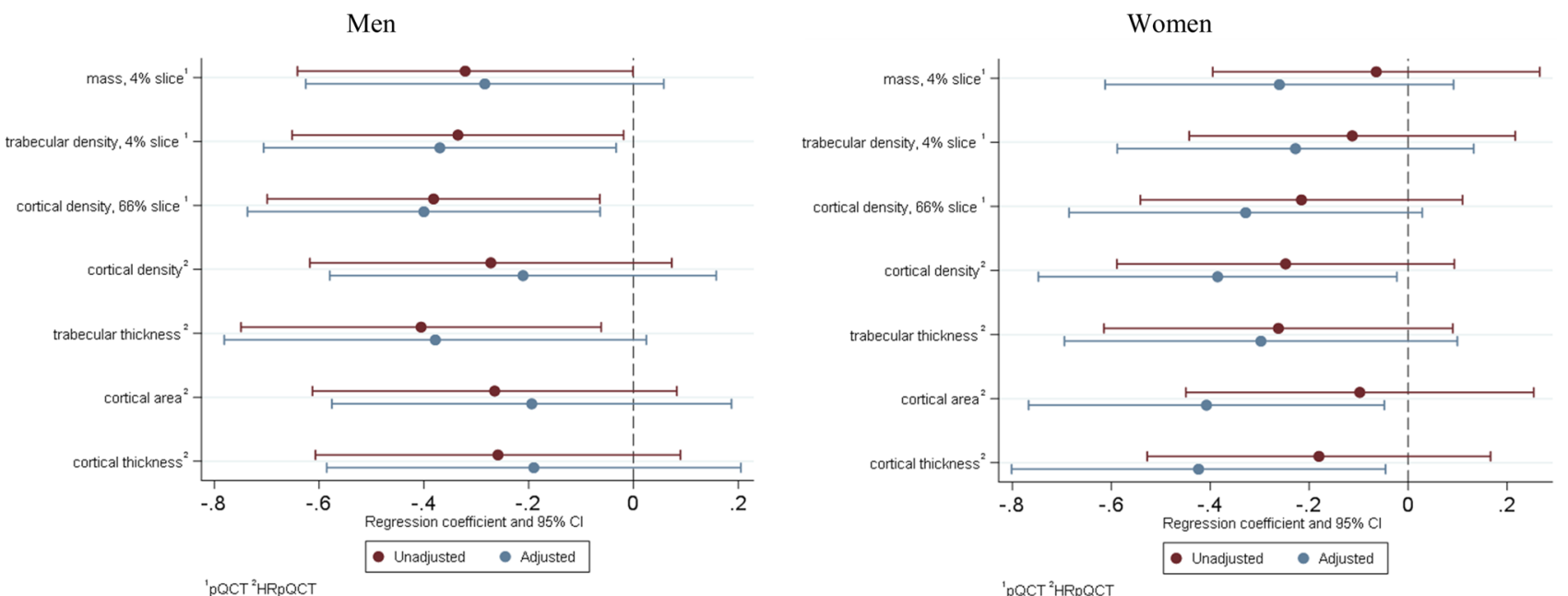

Fig. 1 Linear regression results assessing relationship between PSQI > 5, and PQCT and HRpQCT radial bone outcomes 

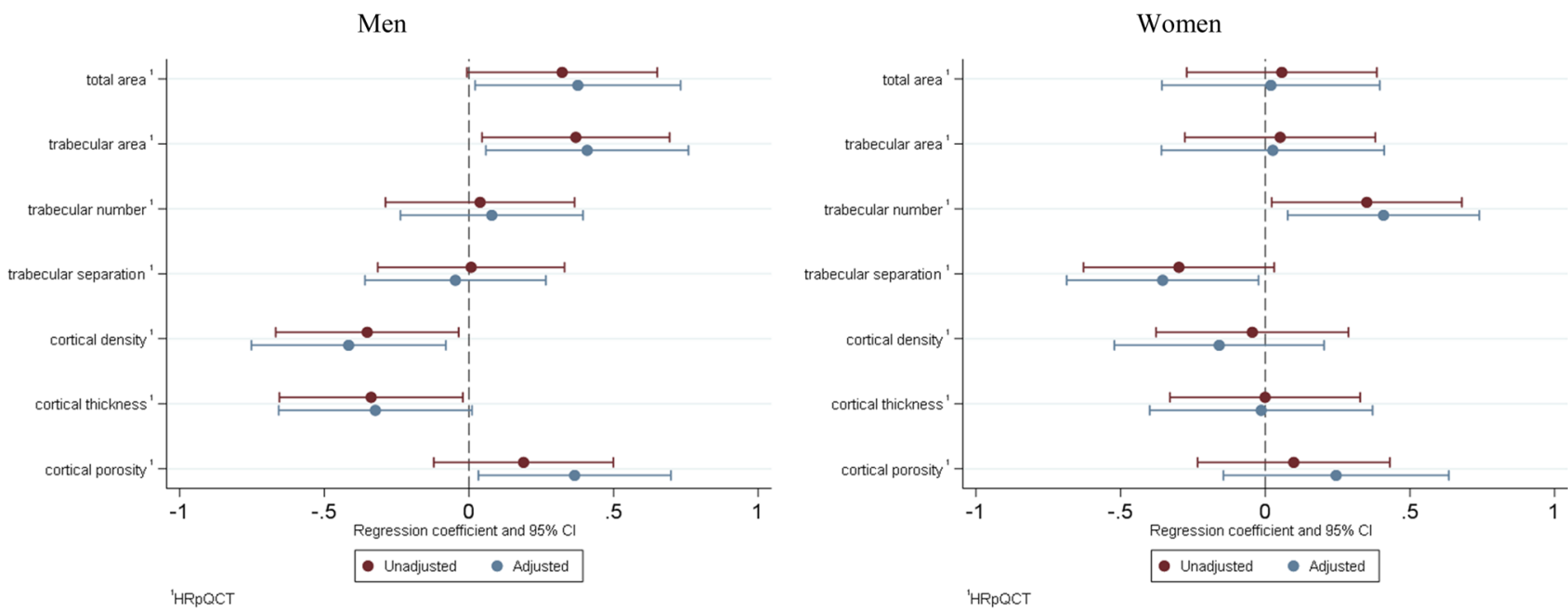

Fig. 2 Linear regression results assessing relationship between PSQI $>5$ and HRpQCT tibial bone outcomes

tibial apparent cortical thickness were attenuated, whilst all other associations remained significant. In addition, associations between poor sleep and higher tibial $\operatorname{Tt} . \operatorname{Ar}(\beta 0.38$ $z$-score, $95 \%$ CI $0.02,0.73, p<0.04)$ and cortical porosity $(\beta$ $0.37 z$-score, $95 \%$ CI $0.03,0.70 p<0.04$ ) became significant after adjustment for confounders.

By contrast, in women, a PSQI score $>5$ was not associated with any of the pQCT outcomes assessed. Poor sleep quality was associated with a higher tibial trabecular number ( $\beta 0.35 z$-score, 95\% CI 0.02, $0.68, p<0.04)$ in women. The association persisted following adjustment for confounders, years after menopause, and HRT use. In women, a PSQI score $>5$ was associated with lower radial Ct.Ar $(\beta-0.41$ $z$-score, $95 \%$ CI $-0.77,-0.05, p<0.03)$, lower cBMD $(\beta$ $-0.39 z$-score, $95 \%$ CI $-0.75,-0.02, p<0.04)$, apparent cortical thickness $(\beta-0.42 z$-score, $95 \%$ CI $-0.80,-0.05$, $p<0.03)$ and lower tibial trabecular separation $(\beta-0.35$ $z$-score, $95 \%$ CI $-0.69,-0.02, p<0.04)$, only after adjustment for confounders and sex-specific confounders.

We also analysed the relationship between individual PSQI subcomponents and bone health outcomes.

\section{Subjective sleep quality}

Poor subjective sleep quality was associated with reduced tibial polar SSI at $66 \%$ slice in men $(\beta-0.22 z$-score, $95 \%$ CI $-0.44,-0.00, p<0.05)$, although the association no longer remained significant following adjustment for confounders. No further association was found in men. Conversely, PSQI poorer subjective sleep quality was associated with lower radial Tt.Ar $(\beta-0.30 z$-score, 95\% CI -0.56 , $-0.03, p=0.03)$ and trabecular area $(\beta-0.30 z$-score, $95 \%$ $\mathrm{CI}-0.56,-0.03, p=0.03$ ) at $4 \%$ slice in women (adjusted results).

\section{Sleep latency}

Similarly, longer sleep latency did not appear to be related to bone health outcomes in men, but was associated with higher tibial trabecular number $(\beta 0.28 z$-score, 95\% CI $0.10,0.46, p=0.003)$ and lower trabecular separation $(\beta-0.26 z$-score, 95\% CI $-0.44,-0.08, p=0.005)$ in women (adjusted results).

\section{Sleep duration}

Following adjustment for confounders and sex-specific confounders, shorter sleep duration was associated with increased tibial cortical porosity in men $(\beta 0.24 z$-score, $95 \%$ CI $0.03,0.44, p<0.03)$ and lower tibial cBMD at $38 \%$ slice in women $(\beta-0.24 z$-score, $95 \% \mathrm{CI}-0.44,-0.04$, $p<0.02)$.

\section{Sleep efficiency}

Poorer habitual sleep efficiency was associated with lower radial mass $(\beta-0.16 z$-score, $95 \% \mathrm{CI}-0.31,-0.00$, $p<0.05)$ at $4 \%$ slice in men, following adjustment for confounders. In women, poorer habitual sleep efficiency was found to be associated with higher tibial mass at $4 \%$ slice ( $\beta 0.19 z$-score, 95\% CI 0.05, 0.33, $p=0.01)$ and trabecular number ( $\beta 0.22 z$-score, 95\% CI 0.07, 0.37, $p=0.005)$, and decreased trabecular separation $(\beta-0.22 z$-score, $95 \% \mathrm{CI}$ $-0.36,-0.07, p=0.005)$, following adjustment for confounders, years after menopause and HRT use. 


\section{Sleep disturbance}

In men, sleep disturbance was associated with increased radial total density ( $\beta 0.31 z$-score, $95 \%$ CI $0.01,0.61$, $p<0.05)$ at $4 \%$ slice, and tibial cBMD at $38 \%$ slice $(\beta 0.36$ $z$-score, $95 \% \mathrm{CI} 0.06,0.65, p=002)$ and $66 \%$ slice $(\beta 0.33$ $z$-score, $95 \%$ CI $0.02,0.65, p<0.04)$, after adjustment. In women, this PSQI domain was associated with decreased radial cortical porosity ( $\beta-0.39 z$-score, $95 \% \mathrm{CI}-0.76$, $-0.03, p<0.04$ ) following adjustment for confounders and sex-specific confounders.

\section{Sleep medication}

A higher frequency of use of sleep medications was associated with lower tibial SSI $(\beta-0.20 z$-score, $95 \%$ CI -0.41 , $-0.00, p<0.05)$, and Tt.Ar at $38 \%$ slice $(\beta-0.22 z$-score, $95 \% \mathrm{CI}-0.42,-0.01, p<0.04)$ and $66 \%$ slice $(\beta-0.21$ $z$-score, $95 \% \mathrm{CI}-0.42,-0.00, p<0.05)$ in women after adjustment, but no association was found in men. It must be noted that women reported a higher use of sleep medications within a month (13.8\%) than men (8.9\%), although the difference between sexes was not statistically significant $(p=0.167)$.

\section{Daytime dysfunction}

No relationship was found between increased daytime dysfunction and bone health outcomes in neither men nor women, once results had been adjusted for confounders and sex-specific confounders.

\section{Discussion}

We have found a high prevalence of poor sleep quality in a population of older community-dwelling adults, and this was consistent with the age-related deterioration of sleep found in previous studies [24]. We did not find associations between poor sleep quality (PSQI $>5$ ) and DXA-derived areal BMD, but in contrast, we found evidence of crosssectional associations between poor sleep quality (PSQI $>5$ ) and altered bone density, strength and microarchitecture measured by pQCT and HRpQCT in both sexes. In general, these associations were stronger in men than in women. These observations may be explained by previous studies that suggest that body size and composition may be related to sleep parameters $[25,26]$; the imperfect body size correction observed with a $2 \mathrm{D}$ areal technique such as DXA reinforces the use of technology that can fully adjust for body size in $3 \mathrm{D}$ where available. Interestingly, the direction of association appeared different at weight bearing and non-weight bearing sites. Results using pQCT scans showed significant associations in men for radial trabecular density at the epiphysis and radial cortical density at the diaphysis, whereas no association was found in women. HRpQCT imaging showed associations in men for total and trabecular areas, and cortical density and porosity at the metaphysis of the tibia, whereas in women poor reported sleep quality was associated with increased trabecular number and decreased separation at the metaphysis of the tibia, and with decreased cortical area, bone density and thickness at the metaphysis of the radius. In women, adjustment for BMI seemed to have a particular effect on the associations between metaphyseal cortical bone outcomes and poor sleep quality. It is possible that this is due to the complexity of relationship between BMI, menopause timing and sleep quality all of which would impact bone; however, such associations would need confirmation in larger cohorts. Associations between individual PSQI subcomponents and bone health outcomes also varied according to biological sex.

The association between sleep quality and bone outcomes has been previously explained as an effect of sleep deprivation on circadian rhythms and thus the consequent changes that occur in bone metabolism [27]. Disruptions in the circadian clock have indeed been linked to abnormal bone metabolism and osteoporosis [28]. In our study, bone outcomes changed according to biological sex, and this can be linked to the fact that sleep quantity and quality varies with biological sex [3]. A study by Mallampalli and colleagues found that women tend to have longer sleep latency than men, and report experiences of unrefreshing sleep and insomnia more frequently, whereas obstructive sleep apnoea is more commonly reported by men; this study also found differences in the prevalence of sleep disorders by sex, with narcolepsy and REM behaviour disorder being more common in men, and idiopathic hypersomnia being more prevalent in women [29]. In general, previous studies on sex-related differences in sleep quality report that women experience more sleep difficulties and have a higher predisposition of insomnia than men [30-33].

In our study, we found that women had a higher prevalence of poor PSQI, which is consistent with the findings of the studies mentioned above. Nonetheless, we found stronger associations between poor sleep quality and altered bone parameters in men prior to adjustments. This may reflect higher consumption of alcohol in men than women (though average intake was low in this group), or a different comorbidity profile, that could include a higher prevalence of chronic obstructive sleep apnoea, which might be a feature of obesity. Despite a significant prior literature that suggested women may report worse sleep than men, relationships appear complex. Hence, a previous study looking at sleep quantity and sleep difficulties in a population of approximately 2000 British individuals aged 16 to 93 years, despite confirming the higher prevalence of sleep difficulties 
in women, found that women had better self-reported sleep quality than men [34]. The population studied, however, had a significantly wider age range than our cohort, and hence, heterogeneity may mask important differences by age.

Our findings are consistent with the sex-related differences in bone microarchitecture reported by Amin and colleagues, who suggested that biological sex is associated with different bone parameters and might be site-specific [35]. In particular, the association in men between poor sleep quality and reduced tibial Tt.Ar and trabecular area (but not number), lower cBMD in both radius and tibia and increased tibial cortical porosity (but neither thickness nor area) are in line with that observed in the above-mentioned study, in which it is reported that men tend to have more trabecular thinning rather than dropout, and experience greater cortical porosity but less cortical thinning than women [35].

However, some of our results were unexpected. Previous literature has suggested that poor sleep may be detrimental to bone health. However, many studies have been performed in night workers where circadian rhythms are significantly disrupted [36-41]. Our study sample is very different; we recruited older men and women who were in retirement and where such considerations were not relevant, though comorbidity burden was higher. Additionally, we have relied on a validated self-reported sleep quality tool. It is important to recognise previous work that has suggested correlations between questionnaire scores and objective measures of sleep quantity are, at best, modest [42]. The variation in relationships by site was interesting, and may represent residual confounding, which might include some aspect of physical activity not captured by our questionnaire. In this study, physical activity was calculated as the average minutes per day spent walking, cycling, gardening, playing sport and doing house work in the last two weeks. It is possible that this approach is too granular, and an appreciation of relationships between certain types of activity to sleep and bone health would be beneficial.

We found that different subcomponents of the PSQI correlated with different pQCT and HRpQCT parameters on specific sites. Although the pattern that emerged seemed to mirror our findings from the overall PSQI score, we found more associations in women rather than men. Further refinement of the PSQI tool may be helpful to dissect relationships further. We were struck by stronger association between the use of sleep medications, and decreased bone strength and size of the tibia in women rather than men. Several central nervous system-active drugs have been associated with an increased risk of fractures [43-45], but only a few studies addressed the possible effect of such drugs on bone mineral density. A cross-sectional cohort study conducted in the US, for instance, found a correlation between decreased bone mineral density and use of anticonvulsants and opioids, but not benzodiazepines and antidepressants [46]. This study's population was however significantly younger than ours. On the other hand, a longitudinal cohort study including more than 2000 elderly Japanese participants (mostly women) found that individuals taking angiotensin converting enzyme inhibitors or benzodiazepines experienced a higher annual loss of bone mineral density than individuals who did not take these drugs [47].

Our study has a number of strengths. HRpQCT provides measurements of bone microarchitecture and has not been previously used to explore the relationship between bone health and sleep quality. A significant strength of this study is the reasonably large sample size in a population of older adults still living in their own homes that have been extensively phenotyped and well characterised with regard to lifestyle and past medical history. Our study has also a number of limitations. The use of a self-report tool for sleep has been discussed previously, although the PSQI has been proven to be a reliable tool in differentiating good from poor sleepers [19]. The study population may not be entirely representative of the wider UK population, since all recruited participants were born in the county of Hertfordshire, were still living there in their eighth decade and were all Caucasian and therefore do not reflect the diversity of other populations in the UK. However, we have previously demonstrated that this cohort is representative of the general population with regard to anthropometric body build and lifestyle factors, such as smoking, alcohol intake and dietary calcium intake, which was in line with data found in the European Investigation into Cancer and Nutrition (EPIC) cohort [48], therefore suggesting that selection bias was minimal [17]. In addition, a 'healthy' responder bias is evident within the HCS [17]; however, it is unlikely to have affected the observed associations between sleep quality and bone parameters. An additional limitation of this study is the fact that sleep quality, as well as other variants, was only measured at one time point. Future studies may benefit from exploring whether longitudinal change in sleep quality is associated with change in bone health.

\section{Conclusion}

We found that quality of sleep was associated with altered bone parameters in a cohort of older community-dwelling adults in the UK. We saw that these relationships differed according to biological sex and site: in men, poor reported sleep quality was associated, with lower tBMD and cBMD of distal and mid-shaft radius, respectively, reduced cortical density and increased cortical porosity of the tibia, but increased size of the tibial total and trabecular areas; in women, poor sleep quality was associated with cortical thinning and loss of BMD of the radius but improved trabecular parameters of the tibia. Taken together, our results suggest 
that self-report of poor sleep may be associated with adverse bone outcomes more so at the radius than tibia, where results were more mixed; consideration of important lifestyle factors such as alcohol consumption, physical activity and comorbidity burden is thus warranted. Distinct PSQI subcomponents seemed to have an effect on specific sites and parameters, and it is difficult to find a physiological explanation for these associations. Future studies might benefit from investigating these associations in larger populations. Use of sleep medication in women was associated with worse bone health and should be considered when reviewing older patients.

Acknowledgements We are extremely grateful to the Hertfordshire Study Cohort participants who took part in each stage of this research, Versus Arthritis, the Medical Research Council of Great Britain and the Porticus Foundation funded the study. Kate A. Ward's research is part-supported by MRC Programme Number U105960371.

Author Contributions GB prepared the first draft of the paper and coordinated all revisions. CC is guarantor. HJD and EMD designed the study and together with $\mathrm{KW}$ oversaw data collection. KAJ was responsible for statistical analysis of the data. All authors reviewed the paper critically for intellectual content and approved the final version. All authors agree to be accountable for the work and to ensure that any questions relating to the accuracy and integrity of the paper are investigated and properly resolved.

\section{Compliance with ethical standards}

Conflict of interest C. Cooper reports personal fees from Alliance for Better Bone Health, Amgen, Eli Lilly, GSK, Medtronic, Merck, Novartis, Pfizer, Roche, Servier, Takeda and UCB. G. Bevilacqua, H. Denison, F. Laskou, K. A. Jameson, K. A. Ward and E. M. Dennison have no relevant interests to declare.

Human and Animal Rights and Informed Consent Ethical approval was granted by the Hertfordshire Research Ethics Committee, reference number 10/H0311/59. All participants fully consented prior to undertaking the study.

Open Access This article is licensed under a Creative Commons Attribution 4.0 International License, which permits use, sharing, adaptation, distribution and reproduction in any medium or format, as long as you give appropriate credit to the original author(s) and the source, provide a link to the Creative Commons licence, and indicate if changes were made. The images or other third party material in this article are included in the article's Creative Commons licence, unless indicated otherwise in a credit line to the material. If material is not included in the article's Creative Commons licence and your intended use is not permitted by statutory regulation or exceeds the permitted use, you will need to obtain permission directly from the copyright holder. To view a copy of this licence, visit http://creativecommons.org/licenses/by/4.0/.

\section{References}

1. Kline C (2013) Sleep quality. In: Gellman MD, Turner JR (eds) Encyclopedia of behavioral medicine. Springer, New York, pp 1811-1813. https://doi.org/10.1007/978-1-4419-1005-9_849

2. Krystal AD, Edinger JD (2008) Measuring sleep quality. Sleep Med 9(Suppl 1):S10-17. https://doi.org/10.1016/s1389 -9457(08)70011-x

3. Redline S, Kirchner HL, Quan SF, Gottlieb DJ, Kapur V, Newman A (2004) The effects of age, sex, ethnicity, and sleep-disordered breathing on sleep architecture. Arch Intern Med 164(4):406-418. https://doi.org/10.1001/archinte.164.4.406

4. Swanson CM, Shea SA, Wolfe P, Cain SW, Munch M, Vujovic N, Czeisler CA, Buxton OM, Orwoll ES (2017) Bone turnover markers after sleep restriction and circadian disruption: a mechanism for sleep-related bone loss in humans. J Clin Endocrinol Metab 102(10):3722-3730. https://doi.org/10.1210/jc.2017-01147

5. Wang D, Ruan W, Peng Y, Li W (2018) Sleep duration and the risk of osteoporosis among middle-aged and elderly adults: a dose-response meta-analysis. Osteoporosis Int 29(8):1689-1695. https://doi.org/10.1007/s00198-018-4487-8

6. Kobayashi D, Takahashi O, Deshpande GA, Shimbo T, Fukui $\mathrm{T}$ (2012) Association between osteoporosis and sleep duration in healthy middle-aged and elderly adults: a large-scale, crosssectional study in Japan. Sleep Breathing 16(2):579-583. https:// doi.org/10.1007/s11325-011-0545-6

7. Chen G, Chen L, Wen J, Yao J, Li L, Lin L, Tang K, Huang H, Liang J, Lin W, Chen H, Li M, Gong X, Peng S, Lu J, Bi Y, Ning G (2014) Associations between sleep duration, daytime nap duration, and osteoporosis vary by sex, menopause, and sleep quality. J Clin Endocrinol Metab 99(8):2869-2877. https://doi.org/10.1210/ jc.2013-3629

8. Cunningham TD, Di Pace BS (2015) Is self-reported sleep duration associated with osteoporosis? Data from a 4-year aggregated analysis from the national health and nutrition examination survey. J Am Geriatr Soc 63(7):1401-1406. https://doi.org/10.1111/ jgs. 13477

9. Tian Y, Shen L, Wu J, Xu G, Yang S, Song L, Zhang Y, Mandiwa C, Yang H, Liang Y, Wang Y (2015) Sleep duration and timing in relation to osteoporosis in an elderly Chinese population: a cross-sectional analysis in the Dongfeng-Tongji cohort study. Osteoporosis Int 26(11):2641-2648. https://doi.org/10.1007/ s00198-015-3172-4

10. Moradi S, Shab-Bidar S, Alizadeh S, Djafarian K (2017) Association between sleep duration and osteoporosis risk in middleaged and elderly women: a systematic review and meta-analysis of observational studies. Metabolism 69:199-206. https://doi. org/10.1016/j.metabol.2017.01.027

11. Chen YL, Weng SF, Shen YC, Chou CW, Yang CY, Wang JJ, Tien KJ (2014) Obstructive sleep apnea and risk of osteoporosis: a population-based cohort study in Taiwan. J Clin Endocrinol Metab 99(7):2441-2447. https://doi.org/10.1210/jc.2014-1718

12. Chakhtoura M, Nasrallah M, Chami H (2015) Obstructive sleep apnea and osteoporosis risk. J Clin Sleep Med 11(9):1071-1072. https://doi.org/10.5664/jcsm.5034

13. Upala S, Sanguankeo A, Congrete S (2015) Obstructive sleep apnea is not associated with an increased risk of osteoporosis: a systematic review and meta-analysis. J Clin Sleep Med 11(9):1069-1070. https://doi.org/10.5664/jcsm.5032

14. Liguori C, Mercuri NB, Izzi F, Romigi A, Cordella A, Piccirilli E, Viola S, Costa S, Sbraccia P, Marciani MG, Tarantino U, Placidi F (2016) Obstructive sleep apnoea as a risk factor for osteopenia and osteoporosis in the male population. Eur Respir J 47(3):987-990. https://doi.org/10.1183/13993003.01830-2015 
15. Upala S, Sanguankeo A, Congrete S (2016) Association between obstructive sleep apnea and osteoporosis: a systematic review and meta-analysis. Int J Endocrinol Metab 14(3):e36317. https://doi. org/10.5812/ijem.36317

16. Liguori C, Piccirilli E, Izzi F, Mercuri NB, Tarantino U, Placidi F (2017) Obstructive sleep apnoea as a risk factor for osteopenia and osteoporosis in the male population: further data and comments. Eur Respir J 49:4. https://doi.org/10.1183/13993003.02471-2016

17. Syddall H, Aihie Sayer A, Dennison E, Martin H, Barker D, Cooper C, Group atHCS (2005) Cohort profile: the hertfordshire cohort study. Int J Epidemiol 34(6):1234-1242. https://doi. org/10.1093/ije/dyi127

18. Syddall H, Simmonds S, Carter S, Robinson S, Dennison E, Cooper C, Null N (2019) The hertfordshire cohort study: an overview [version 1; peer review: 3 approved]. F1000Research 8:82. https://doi.org/10.12688/f1000research.17457.1

19. Buysse DJ, Reynolds CF 3rd, Monk TH, Berman SR, Kupfer DJ (1989) The Pittsburgh Sleep Quality Index: a new instrument for psychiatric practice and research. Psychiatry Res 28(2):193-213

20. Shaw SC, Parsons CM (2018) Diet quality and bone measurements using HRpQCT and pQCT in older community-dwelling adults from the hertfordshire cohort study. Calcified Tissue Int 103(5):494-500. https://doi.org/10.1007/s00223-018-0445-x

21. Boutroy S, Bouxsein ML, Munoz F, Delmas PD (2005) In vivo assessment of trabecular bone microarchitecture by high-resolution peripheral quantitative computed tomography. J Clin Endocrinol Metab 90(12):6508-6515. https://doi.org/10.1210/jc.2005-1258

22. Bingham SA, Gill C, Welch A, Day K, Cassidy A, Khaw KT, Sneyd MJ, Key TJ, Roe L, Day NE (1994) Comparison of dietary assessment methods in nutritional epidemiology: weighed records $v 24 \mathrm{~h}$ recalls, food-frequency questionnaires and estimated-diet records. Br J Nutr 72(4):619-643. https://doi.org/10.1079/bjn19940064

23. Robinson SM, Batelaan SF, Syddall HE, Sayer AA, Dennison EM, Martin HJ, Barker DJ, Cooper C (2006) Combined effects of dietary fat and birth weight on serum cholesterol concentrations: the Hertfordshire Cohort Study. Am J Clin Nutr 84(1):237-244. https://doi. org/10.1093/ajcn/84.1.237

24. Ohayon MM, Carskadon MA, Guilleminault C, Vitiello MV (2004) Meta-analysis of quantitative sleep parameters from childhood to old age in healthy individuals: developing normative sleep values across the human lifespan. Sleep 27(7):1255-1273. https://doi.org/10.1093/ sleep/27.7.1255

25. Eleuteri S, Norton MC, Livi F, Grano C, Falaschi P, Violani C, Lucidi F, Lombardo C (2018) Sleep quality as predictor of BMI in non-depressed caregivers of people with dementia. Eating Weight Disorders 23(5):553-560. https://doi.org/10.1007/s4051 9-018-0560-8

26. Bayon V, Leger D, Gomez-Merino D, Vecchierini MF, Chennaoui M (2014) Sleep debt and obesity. Ann Med 46(5):264-272. https:// doi.org/10.3109/07853890.2014.931103

27. Specker BL, Binkley T, Vukovich M, Beare T (2007) Volumetric bone mineral density and bone size in sleep-deprived individuals. Osteoporos Int 18(1):93-99. https://doi.org/10.1007/s0019 8-006-0207-X

28. Song C, Wang J, Kim B, Lu C, Zhang Z, Liu H, Kang H, Sun Y, Guan H, Fang Z (2018) Insights into the role of circadian rhythms in bone metabolism: a promising intervention target? BioMed Res Int. https://doi.org/10.1155/2018/9156478

29. Mallampalli MP (2002) Carter CL (2014) Exploring sex and gender differences in sleep health: a Society for Women's Health Research Report. J Women's Health 23(7):553-562. https://doi.org/10.1089/ jwh.2014.4816

30. Middelkoop HA, Smilde-van den Doel DA, Neven AK, Kamphuisen HA, Springer CP (1996) Subjective sleep characteristics of 1485 males and females aged 50-93: effects of sex and age, and factors related to self-evaluated quality of sleep. J Gerontol Ser A 51(3):M108-115

31. Lindberg E, Janson C, Gislason T, Bjornsson E, Hetta J, Boman G (1997) Sleep disturbances in a young adult population: can gender differences be explained by differences in psychological status? Sleep 20(6):381-387

32. Zhang B, Wing YK (2006) Sex differences in insomnia: a metaanalysis. Sleep 29(1):85-93

33. Mong JA, Cusmano DM (2016) Sex differences in sleep: impact of biological sex and sex steroids. Philos Trans R Soc Lond B 371(1688):20150110-20150110. https://doi.org/10.1098/ rstb.2015.0110

34. Groeger JA, Zijlstra FR, Dijk DJ (2004) Sleep quantity, sleep difficulties and their perceived consequences in a representative sample of some 2000 British adults. J Sleep Res 13(4):359-371. https://doi. org/10.1111/j.1365-2869.2004.00418.x

35. Amin S, Khosla S (2012) Sex- and age-related differences in bone microarchitecture in men relative to women assessed by high-resolution peripheral quantitative computed tomography. J Osteoporosis 2012:129760. https://doi.org/10.1155/2012/129760

36. Boivin DB, Boudreau P (2014) Impacts of shift work on sleep and circadian rhythms. Pathologie-biologie 62(5):292-301. https://doi. org/10.1016/j.patbio.2014.08.001

37. Jin Y, Hur TY, Hong Y (2017) Circadian rhythm disruption and subsequent neurological disorders in night-shift workers. J Lifestyle Med 7(2):45-50. https://doi.org/10.15280/jlm.2017.7.2.45

38. Hunter CM, Figueiro MG (2017) Measuring light at night and melatonin levels in shift workers: a review of the literature. Biol Res Nursing 19(4):365-374. https://doi.org/10.1177/1099800417714069

39. Manodpitipong A, Saetung S, Nimitphong H, Siwasaranond N, Wongphan T, Sornsiriwong C, Luckanajantachote P, Mangjit P, Keesukphan P, Crowley SJ, Hood MM, Reutrakul S (2017) Nightshift work is associated with poorer glycaemic control in patients with type 2 diabetes. J Sleep Res 26(6):764-772. https://doi. org/10.1111/jsr.12554

40. Morris CJ, Purvis TE, Mistretta J, Hu K, Scheer F (2017) Circadian misalignment increases $\mathrm{C}$-reactive protein and blood pressure in chronic shift workers. J Biol Rhythms 32(2):154-164. https://doi. org/10.1177/0748730417697537

41. Bukowska-Damska A, Skowronska-Jozwiak E, Peplonska B (2019) Night shift work and osteoporosis: evidence and hypothesis. Chronobiol Int 36(2):171-180. https://doi.org/10.1080/07420 528.2018.1528553

42. O'Brien E, Hart C, Wing RR (2016) Discrepancies between selfreported usual sleep duration and objective measures of total sleep time in treatment-seeking overweight and obese individuals. Behav Sleep Med 14(5):539-549. https://doi.org/10.1080/15402 002.2015 .1048447

43. Vestergaard P (2008) Skeletal effects of central nervous system active drugs: anxiolytics, sedatives, antidepressants, lithium and neuroleptics. Curr Drug Safety 3(3):185-189

44. Lin F-Y, Chen P-C, Liao CH, Hsieh Y-W, Sung F-C (2014) Retrospective population cohort study on hip fracture risk associated with zolpidem medication. Sleep 37(4):673-679. https://doi.org/10.5665/ sleep.3566

45. Tang Y-J, Ho S-Y, Chu F-Y, Chen H-A, Yin Y-J, Lee H-C, Chu WC-C, Yeh H-W, Chiang W-S, Yeh C-L, Huang H-L, Tzeng N-S (2015) Is zolpidem associated with increased risk of fractures in the elderly with sleep disorders? A nationwide case cross-over study in Taiwan. PLoS ONE 10(12):e0146030-e0146030. https://doi. org/10.1371/journal.pone.0146030

46. Kinjo M, Setoguchi S, Schneeweiss S, Solomon DH (2005) Bone mineral density in subjects using central nervous system-active medications. Am J Med 118(12):1414. https://doi.org/10.1016/j. amjmed.2005.07.033 
47. Masunari N, Fujiwara S, Nakata Y, Furukawa K, Kasagi F (2008) Effect of angiotensin converting enzyme inhibitor and benzodiazepine intake on bone loss in older Japanese. Hiroshima J Med Sci 57(1):17-25

48. Dik VK, Murphy N, Siersema PD, Fedirko V, Jenab M, Kong SY, Hansen CP, Overvad K, Tjonneland A, Olsen A, Dossus L, Racine A, Bastide N, Li K, Kuhn T, Boeing H, Aleksandrova K, Trichopoulou A, Trichopoulos D, Barbitsioti A, Palli D, Contiero P, Vineis P, Tumino R, Panico S, Peeters PH, Weiderpass E, Skeie G, Hjartaker A, Amiano P, Sanchez MJ, Fonseca-Nunes A, Barricarte A, Chirlaque MD, Redondo ML, Jirstrom K, Manjer J, Nilsson LM, Wennberg M, Bradbury KE, Khaw KT, Wareham N, Cross AJ, Riboli E,
Bueno-de-Mesquita HB (2014) Prediagnostic intake of dairy products and dietary calcium and colorectal cancer survival-results from the EPIC cohort study. Cancer Epidemiol Biomarkers Prev 23(9):1813-1823. https://doi.org/10.1158/1055-9965.epi-14-0172

Publisher's Note Springer Nature remains neutral with regard to jurisdictional claims in published maps and institutional affiliations. 